\title{
Lípidos, sodio y fibra dietética en harina de trigo y pan artesanal en Uruguay: aporte nutricional según recomendaciones para distintos grupos de población
}

\author{
Lipids, sodium and dietary fiber in wheat \\ flour and artisan bread in Uruguay: \\ nutritional intake according to the \\ recommendations for different population groups
}

\begin{abstract}
ABTRACT
This research assessed the proportion of whole-wheat flour in whole-wheat bread relative to the level mandated by law, as well as the content of lipids (Lip) and sodium ( $\mathrm{Na}$ ) in French bread, and of Lip, Na and total dietary fiber (TDF) in whole-wheat bread made in bakeries in Montevideo and Canelones, Uruguay. The percentage of $\mathrm{Na}$ and TDF using both Adequate Intakes (Als) and Tolerable Upper Intake levels (UIS) were estimated in reference to recommended daily quantities. Na in French bread consists of $64 \%$ of Als for individuals aged nine and older, and over $100 \%$ of Als for adults aged 51 and older. Whole-wheat bread values are slightly lower. TDF consists of $38 \%$ of Als in 9 year-old children and $53 \%$ in adults older than 50 years of age. This work helps to establish a baseline for the development of formulations with minimum levels of $\mathrm{Na}$ and optimum levels of TDF in artisan bread. Key words: Sodium; dietary fiber; Adequate Intakes; Tolerable upper intake levels; artisan bread.
\end{abstract}

\section{INTRODUCCIÓN}

La harina de trigo y los panificados, proveen nutrientes a la alimentación que se relacionan con algunas de las enfermedades crónicas no transmisibles (ECNT) como son las enfermedades cardiovasculares (ECV), obesidad y el cáncer de alta prevalencia en Uruguay como en muchos países del mundo $(1,2)$.

Existe evidencia científica sobre el papel preventivo de la fibra dietética (FD). Por otro las dietas ricas en sodio ( $\mathrm{Na}$ ) y un perfil lipídico particular aumentan el riesgo de ECV (3). Las que constituyen la primer causa de muerte del total de causas de defunción (4). La calidad de la materia prima y la formulación del pan francés e integral artesanales inciden en la composición de $\mathrm{Na}$, lípidos y FD de estos alimentos. El hábito
Marta Elichalt (1)

Mónica Russo (1)

Daniel Vázquez (2)

Gabriela Suburú (3)

HugoTihista (4)

Marcela Godiño (5)

(1) Escuela de Nutrición, Universidad de la República Oriental del Uruguay (2) Instituto Nacional de Investigación Agropecuaria la Estanzuela. Uruguay. (3) Departamento de Cereales, Oleaginosas y Productos Derivados, Laboratorio Tecnológico del Uruguay

4) Instituto Tecnológico del Pan. Centro de Industriales Panaderos del Uruguay (5) Mesa Nacional del Trigo, Uruguay

Dirigir correspondencia a Profesora
Marta Elichalt
Escuela de Nutrición. UdelaR. Paysandú 843.
Montevideo Profesora
Marta Elichalt
Escuela de Nutrición. UdelaR. Paysandú 843.
Montevideo

Montevideo Uruguay Fono: 29008415

Email: melichalt@nutricion.edu.uy

Este trabajo fue recibido el 25 de Mayo de 2016 y aceptado para ser publicado el 16 de Diciembre de 2016.

en el consumo de estos panes artesanales puede por lo tanto, incidir en la alimentación como un factor dietético preventivo o de riesgo para el desarrollo de ECNT.

\section{Factores tecnológicos que inciden en la composición nutricional del pan artesanal}

Para el caso del pan integral el Reglamento Bromatológico Nacional (5), define una relación mínima de un 30\% de harina integral sobre el total de harina empleada en la elaboración como requisito para la denominación de pan integral. La misma fuente no incluye materia grasa en la elaboración de pan francés, mientras que menciona la grasa comestible como ingrediente en el pan integral. En relación a la composición en $\mathrm{Na}$, ensayos que evalúan la influencia de distintas concentraciones 
en la reología de la masa en la elaboración del pan, encontraron que podrían emplearse concentraciones entre 1,4\% a 1,6\%, ya que las masas no presentan diferencias en las características plásticas, a la vez que son las concentraciones preferidas por los consumidores (6). Esto supondría una reducción de entre $36 \%$ al $27 \%$ si se partiera de una concentración de 2,2\% de sal en relación a la harina como menciona el estudio.

Composición nutricional de pan artesanal

La falta de datos de composición nutricional en FD en harina integral, en Na y lípidos en pan francés artesanal, así como de $\mathrm{Na}$, lípidos y FD en pan integral artesanal son una limitante en el establecimiento de estrategias preventivas para algunas ECNT como las mencionadas. En un estudio realizado en Chile (2010) por el Ministerio de Salud (7), el contenido de $\mathrm{Na}$ en 4 variedades de pan mostró una concentración promedio de $774 \pm 105 \mathrm{mg}$ cada $100 \mathrm{~g}$, con nivel mayor en marraquetas de $831 \mathrm{mg} \pm 105$ cada $100 \mathrm{~g}$ de alimento. En 2013. se encontró en panes provenientes de panaderías adheridas, un contenido en Na que osciló entre 497 y 824 mg cada 100 g y algo menos fue la concentración en el grupo control (panaderías no adheridas) 403 y $768 \mathrm{mg}$ (7).

En Argentina (2010), el Ministerio de Salud de la Nación, en conjunto con la Federación Argentina de la industria de pan y afines (FAIPA) y el Instituto de Tecnología Industrial (INTI), acordaron disminuir a un 1,5\% el agregado de sal en la formulación de pan (8). En Uruguay (2013), el Ministerio de Salud, la Comisión Honoraria de Salud Cardiovascular y el Centro de Industriales Panaderos del Uruguay (CIPU), firmaron un acuerdo para la reducción voluntaria y progresiva del contenido de $\mathrm{Na}$ en los panificados $(9,10)$. Se parte de una base de adición de un $2 \%$ de sal en relación a la harina y se considera que se puede ir reduciendo en forma paulatina exhortando a una reducción del 10\% y llegar como máximo al $1,8 \%$ de sal en relación a la harina en la formulación del pan. Se ha establecido como meta $400 \mathrm{mg} / 100$ de $\mathrm{Na}$ en el alimento listo para el consumo.

Respecto al contenido de FD de harina de trigo, algunos estudios revelan un tenor promedio de 15,6 \pm 0,12 g por cada $100 \mathrm{~g}$ de alimento (11). Para el pan integral, la información proviene de pan integral industrializado. Según la rotulación el contenido promedio de fibra en 13 productos comercializados en Uruguay cuyas denominaciones corresponden a "pan integral o pan de salvado" fue de $6,7 \mathrm{~g}$ cada $100 \mathrm{~g}$, oscilando entre 4,6 g y 9,3 g en $100 \mathrm{~g}$ de alimento (12).

\section{Ingesta y Recomendaciones nutricionales de $\mathrm{Na}$, FDT y Lípidos}

En promedio, el consumo de sal $(\mathrm{NaCl})$ a nivel mundial bordea el doble de lo recomendado, con valores que fluctúan entre los 9 y $12 \mathrm{~g}$ diarios y para nuestro continente 11,5 g (9). Según datos indirectos de consumo de panes artesanales que surgen de la Encuesta Nacional de Gastos e Ingresos de los Hogares (2005-2006) en Uruguay (13) el grupo de productos panificados fue el que aportó mayor $\mathrm{Na}$ a la dieta. Solo es igualado por otros grupos en el mayor nivel de ingresos (14).

El Ministerio de Salud de Uruguay establece dentro de las metas nutricionales para la población uruguaya (15) en nutrientes críticos para la prevención de algunas ECNT, un 25\% a $30 \%$ de grasa total de la energía de la dieta y menos de 2 g/día de Na (menos de 5g/d de sal) (10). La OMS (2013), en las directrices para la ingesta de $\mathrm{Na}$ (16), recomienda rebajar en los adultos el consumo de Na por debajo de los $2 \mathrm{~g}$ al día, a fin de reducir la tensión arterial y el riesgo de cardiopatía coronaria. En el caso de los niños, el consumo de Na debería ser menor al valor de los adultos para que sea proporcional a las necesidades energéticas. Según información publicada por The National Institutes of Health en USA, no se puede establecer el requerimiento promedio estimado y por ende tampoco obtener su RDA (Recommended Dietary Allowance), debido a la falta de estudios rigurosos (17). Ellos proponen una Ingesta Adecuada (IA) de sal, de 3 g para niños; 3,8 g para adultos menores de 50 años y entre 3,0 y 3,3 g para mayores de esa edad. Por su parte los límites máximos tolerables (ULs) para los niños son de 3,8 a 4,8 g y 5,8 g para el resto de las edades (17).

En el caso de la FD, las recomendaciones varían según diferentes fuentes, van desde 18 a 38 g/día. La OMS y la Autoridad Europea de Seguridad Alimentaria (EFSA), recomiendan 25 g/día. En Canadá, las recomendaciones son de 25 g/día para mujeres y 38 g/día para hombres (18). La Asociación Americana de Dietistas recomienda para adultos sanos entre 20 a 35 g/día (19). Para la Academia Nacional de Ciencias de los EEUU, la Ingesta Adecuada (IA) de fibra total (suma de fibra dietética y funcional) varía según sexo y para diferentes grupos de edad y oscila entre $19 \mathrm{~g} /$ día para niños de 1 a 3 años hasta 38 g/día para hombres de 14 a 50 años (20). Estas últimas se basan en el consumo medio de fibra en la dieta de la población y la reducción del riesgo de ECV en estudios de cohorte. Las mismas consideran un aporte de 14 g fibra cada $1000 \mathrm{Kcal}$. Otras fuentes consideran para estimar las necesidades de fibra en niños, adicionar $5 \mathrm{~g}$ de fibra al valor de edad en años que tenga el niño (21). Con esta fórmula a los 18 años se alcanzaría la cantidad adecuada en la ingesta.

Este estudio tuvo como propósito generar información sobre la composición nutricional de panes artesanales (francés e integral). Estos datos permiten estimar el aporte diario de $\mathrm{Na}$, FDT y lípidos con el consumo de pan artesanal para diferentes grupos de población suponiendo su inclusión en cantidades adecuadas a una alimentación saludable. Además contribuye a establecer una línea de base de composición nutricional en panes artesanales que permita para valorar el cumplimiento del compromiso interinstitucional en la reducción de $\mathrm{Na}$ y sugerir formulaciones estándares con perfiles saludables. Por último permite educar a la población en el valor nutricional de estos alimentos según las recomendaciones de una alimentación saludable con énfasis en la prevención de ECNT.

Los objetivos de este estudio fueron: evaluar el cumplimiento de la legislación nacional respecto a la proporción de harina integral empleada para elaborar pan integral; valorar el contenido de lípidos y $\mathrm{Na}$ en pan francés artesanal y lípidos, $\mathrm{Na}$ y FDT (suma de fibra dietética soluble e insoluble) en pan integral elaborado en panaderías de Montevideo y Canelones; estimar el porcentaje de adecuación a las recomendaciones (Als) y los límites máximos tolerables (ULs) de $\mathrm{Na}$ en pan francés e integral y Al de FDT en pan integral artesanal, en diferentes grupos de población y con diferente concentración de los nutrientes de estudio (mínimo, mediana, máximo) a partir de una ingesta diaria de pan acorde a una alimentación saludable.

\section{MATERIAL Y METODOS}

Muestras y método de recolección de datos

El estudio se llevó a cabo en las ciudades de Montevideo y Canelones, analizando diez muestras de harinas blanca e integral provenientes cada una de cinco molinos (dos lotes distintos por molino) y diez muestras de panes franceses e integrales elaborados en diez panaderías diferentes. Se selec- 
cionaron molinos con diferente capacidad de producción y que en su conjunto abastecieran al 75\% del mercado interno del país. Las muestras de harina se extrajeron en las panaderías seleccionadas a partir de dos lotes diferentes. Se retiró $1 \mathrm{~kg}$ de la parte superior, del medio y de la parte inferior de una bolsa de harina en cada lote. Las muestras obtenidas se embolsaron herméticamente para su protección y estabilidad.

El pan se obtuvo de panaderías seleccionadas en conveniencia con el CIPU entre los asociados de Montevideo y Canelones. Estas panaderías fueron abastecidas con harina de los lotes analizados.

El pan francés se elaboró a partir de la harina proveniente de la misma bolsa que se utilizó para generar las muestras de harina, siguiendo la formulación y el proceso de elaboración habitual de las panaderías. Inmediatamente a su cocción se retiraron tres panes de la bandeja superior, tres del medio y tres de la parte inferior del horno; se acondicionaron en bolsas herméticas y se congelaron a $-18^{\circ} \mathrm{C}$. Las muestras colectadas se homogenizaron previamente a su determinación.

Determinación de la cantidad Lípidos,

$\mathrm{Na}, \mathrm{FD}$ en harina integral y pan francés

La determinación de lípidos se realizó por extracción con éter de petróleo usando aparato Soxhlet (Protocolo: PEC. AYE.003/ITR.004_SOXHLET). La proporción empleada de harina de trigo integral en la formulación así como el tipo de materia grasa, se obtuvo por declaración del maestro panadero de las panaderías incluidas para el estudio.

La determinación de FDT por Norma AOAC 985.29; Fibra Soluble Norma AOAC991.42. La determinación de Na se realizó tanto en harina blanca como integral por emisión (ICP-OES segun ISO 11885:2007 adaptado) y el pan por absorción por atomización por llama aire-acetileno (FAAS) según método AOAC 973.54:2007.

Análisis de datos

Para evaluar la proporción de harina integral empleada para elaborar pan integral con el nivel legislado, se calculó la proporción de harina integral empleada en la formulación según la declaración de los panaderos. La evaluación en el contenido de lípidos y $\mathrm{Na}$ en pan francés artesanal y lípidos, $\mathrm{Na}$ y FDT en pan integral, se analizó a partir de las determinaciones analíticas y el cálculo del valor de la mediana. Con el fin de estimar el grado de adecuación de las muestras de pan francés e integral estudiadas en Na y FDT en pan integral con las ingestas diarias recomendadas (IAs y ULs) para distintos grupos de la población uruguaya, se consideraron las recomendaciones energéticas para la población uruguaya (15) y se estimó la ingesta/día de pan para diferentes grupos de población en base a una alimentación saludable. Posteriormente se calculó la adecuación de las IAs y Uls de Na y IAs de FDT basándose en dichas ingestas para distintos grupos de población.

\section{RESULTADOS Y DISCUSIÓN}

En la harina integral la mediana de la FDT fue de 12,4 g (tabla 1) y osciló entre 9,3 g y 14,8 g. Se advierte variabilidad entre el valor mínimo y máximo probablemente debido a las diversas proporciones consideradas en las mezclas de harina de trigo. El nivel máximo alcanzado es similar al encontrado en otros estudios (11).

En el pan francés se evidencia que no hay agregado de lípidos en la formulación, ya que la mediana fue de 0,28 g, con un mínimo de 0,13 g y un máximo de 1,08 g cada 100 $\mathrm{g}$ de alimento (tabla 2). Estos valores se corresponden a los lípidos naturales aportados por la harina. Por lo tanto, en las muestras estudiadas se cumplió con la normativa vigente (5); el pan francés es un panificado que no vehicula grasa frente a otros que proveen cantidad y calidad de grasas que constituyen un riesgo para la ECV (3).

Al estudiar el $\mathrm{Na}$ en el pan francés (tabla 2) la mediana fue de $642 \mathrm{mg} /$ cada $100 \mathrm{~g}$ con un valor mínimo de $171 \mathrm{mg}$ y un máximo de $802 \mathrm{mg}$ cada $100 \mathrm{~g}$ de pan. Se observa gran variabilidad en el contenido de $\mathrm{Na}$ entre las muestras estudiadas. Estos valores son similares a los encontrados en Chile donde se ha implementado el programa piloto de reducción de $\mathrm{Na}$ en el pan artesanal (7).

Al estudiar la contribución del pan francés en población con diferente edad y sexo suponiendo un consumo/día ade-

\section{TABLA 1}

Contenido de fibra dietética total (FDT) en harina de trigo integral para elaborar pan integral artesanal en panaderías de Montevideo y Canelones.

\begin{tabular}{|c|c|c|c|}
\hline & & $\begin{array}{l}\text { Harina de trigo integral } \\
\qquad(\mathrm{n}=10)\end{array}$ & \\
\hline Concentración g/100 g harina & Mínimo & Mediana & Máximo \\
\hline FDT & 9,3 & 12,4 & 14,8 \\
\hline
\end{tabular}

\section{TABLA 2}

Contenido lípidos y sodio ( $\mathrm{Na}$ ) en pan francés artesanal.

Pan francés artesanal

$$
(\mathrm{n}=10)
$$

Concentración en $100 \mathrm{~g}$ pan

Lípidos $g$

$\mathrm{Na}$ mg

$\begin{array}{cc}\text { Mínimo } & \text { Mediana } \\ 0,13 & 0,28 \\ 171 & 642\end{array}$
Máximo
1,08
802


TABLA 3

Contenido lípidos y sodio (Na), Fibra Dietética Total (FDT) en pan integral artesanal.

Pan integral artesanal

$$
(\mathrm{n}=10)
$$

Concentración en $100 \mathrm{~g}$ de pan

Lípidos g

Na mg

FDT

Mínimo
0,71
210
4,8

Mínimo

210

Mediana

3,1

540

7,9
Máximo

4,75

750

9,7

\section{TABLA 4}

Estimación (\%) de la adecuación a las recomendaciones (AIs) y los límites máximos tolerables (ULs) de Na con diferentes niveles de concentración de $\mathrm{Na}$ en el pan francés, en distintos grupos de población.

\begin{tabular}{|c|c|c|c|c|c|c|c|}
\hline \multirow[t]{2}{*}{$\begin{array}{l}\text { Edad (años) } \\
\text { y sexo }\end{array}$} & \multirow[t]{2}{*}{$\begin{array}{l}\operatorname{Pan}^{a} \\
\text { (g/día) }\end{array}$} & \multicolumn{3}{|c|}{$\begin{array}{c}\text { (mg/día) } \\
\text { Ingesta Adecuadab }\end{array}$} & \multicolumn{3}{|c|}{$\begin{array}{l}\text { (mg/día) } \\
\text { Nivel Máximob }\end{array}$} \\
\hline & & Mín & Med & Máx & Mín & Med & Max \\
\hline 1 a $3 F M$ & 40 & 6,84 & 25,68 & 32,08 & 4,56 & 17,12 & 21,38 \\
\hline 4 a 8 FM & 75 & 10,68 & 40,12 & 50,12 & 6,75 & 25,34 & 31,65 \\
\hline 9 a 13 FM & 150 & 17,1 & 64,2 & 80,2 & 11,65 & 43,77 & 54,68 \\
\hline 14 a $50 \mathrm{~F}$ & 150 & 17,1 & 64,2 & 80,2 & 11,15 & 41,86 & 52,30 \\
\hline 14 a $50 M$ & 200 & 22,8 & 85,6 & 106,93 & 14,86 & 55,82 & 69,73 \\
\hline 51 a 70 FM & 200 & 26,30 & 98,76 & 123,38 & 14,86 & 55,82 & 69,73 \\
\hline$>70 \mathrm{FM}$ & 150 & 21,37 & 80,25 & 100,25 & & & \\
\hline
\end{tabular}

angesta diaria estimada de pan. Se consideraron las porciones recomendadas para una alimentación saludable según las Guías Alimentarias Basadas en Alimentos para la población uruguaya (MSP, 2005).

'Los valores expresan los porcentajes de la Ingesta Adecuada (IA) de Na y Niveles Máximos (ULs) de Na, para no tener riesgos adversos (Dietary Reference Intakes for Water, Potassium, Sodium, Chloride, and Sulfate. 2004) que son cubiertas por la ingesta diaria estimada de pan para diferentes grupos de población y niveles de concentración de Na.

\section{FIGURA 1}

Estimación (\%) de la adecuación a las recomendaciones (Als) de Na con diferentes niveles de concentración de $\mathrm{Na}$ en el pan francés.

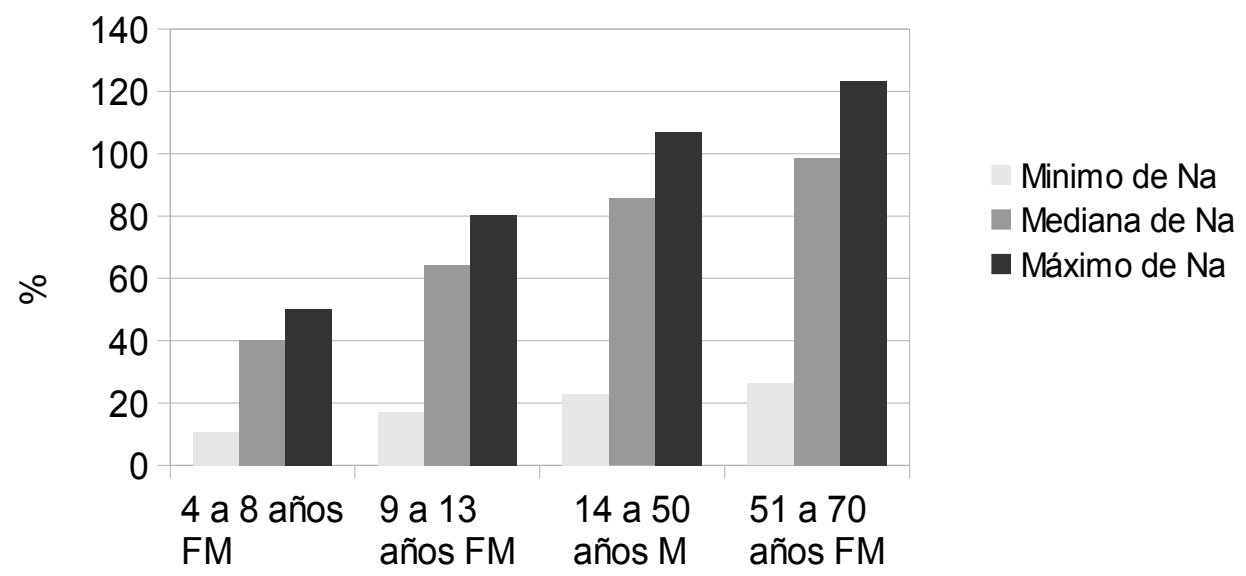


cuado para una alimentación saludable, se observa (tabla 4 , figura 1) que con los valores de la mediana y máximos de $\mathrm{Na}$ del pan francés, a partir de los 9 años, se cubre el $64 \%$ de las IAs y alcanza un valor próximo al 100\% en adultos de 51 a 70 años. Por lo tanto, los valores de adecuación de $\mathrm{Na}$ a las IAs se cubrirían exclusivamente con el pan aun considerando el valor de $\mathrm{Na}$ de la mediana, por lo que es posible alcanzar e incluso superar las IAs sumando el aporte de $\mathrm{Na}$ de otros alimentos de consumo frecuente, más la sal adicionada a las preparaciones. Para un adulto entre 51 a 70 años, al considerar una ingesta diaria de pan de $200 \mathrm{~g}$ para el nivel de la mediana de $\mathrm{Na}$ del pan francés, implicaría un aporte próximo a 1,2 g de $\mathrm{Na}$, lo que equivale a $3 \mathrm{~g}$ de $\mathrm{NaCl}$ solo con el consumo de pan. Es lógico pensar que es fácilmente superable el valor máximo recomendado de $2 \mathrm{~g}$ diarios de $\mathrm{Na}$ o $5 \mathrm{~g}$ de $\mathrm{NaCl}(3$,
15,16). Particularmente en niños, se conoce que entre un $2 \%$ a un $3 \%$ tienen sub-diagnosticada la hipertensión arterial. Es importante considerar que solo con el pan se estaría alcanzando un porcentaje elevado de las IAs de niños mayores de 9 años. Por esta razón se vuelve sustancial que el pan tenga los mínimos niveles de Na posibles en su elaboración y además se propicie una alimentación saludable entre otras medidas promotoras de salud.

En el estudio realizado en Chile, se observa que los valores mínimos y máximos tanto para las panaderías adheridas al Programa de reducción de $\mathrm{Na}$ como en el grupo control (7) son superiores a los encontrados en nuestro estudio.

En este sentido puede ser auspicioso el acuerdo alcanzado por el Ministerio de Salud de Uruguay y el CIPU en 2013 $(9,10)$, ya que se observa que el valor de $400 \mathrm{mg}$ cada $100 \mathrm{~g}$ de

TABLA 5

Estimación (\%) de la adecuación a las recomendaciones (Als) y los límites máximos tolerables (ULs) de Na con diferentes niveles de concentración de $\mathrm{Na}$ en el pan integral, en distintos grupos de población.

\begin{tabular}{|c|c|c|c|c|c|c|c|}
\hline \multirow[t]{2}{*}{$\begin{array}{l}\text { Edad (años) } \\
\text { y sexo }\end{array}$} & \multirow[t]{2}{*}{$\begin{array}{c}\text { Pan }^{\mathrm{a}} \\
\text { (g/día) }\end{array}$} & \multicolumn{3}{|c|}{$\begin{array}{c}\text { (mg/día) } \\
\text { Ingesta Adecuadab }\end{array}$} & \multicolumn{3}{|c|}{$\begin{array}{l}\text { (mg/día) } \\
\text { Nivel Máximob }\end{array}$} \\
\hline & & Mín & Med & Máx & Mín & Med & Max \\
\hline 1 a $3 F M$ & 40 & 8,4 & 21,6 & 30 & 5,6 & 14,4 & 20 \\
\hline 4 a 8 FM & 75 & 13,12 & 33,75 & 46,87 & 8,2 & 21,31 & 29,6 \\
\hline 9 a 13 FM & 150 & 21 & 54 & 75 & 14,3 & 36,81 & 51,13 \\
\hline 14 a $50 \mathrm{~F}$ & 150 & 21 & 54 & 75 & 13,69 & 35,21 & 48,91 \\
\hline 14 a $50 M$ & 200 & 28 & 72 & 100 & 18,26 & 46,95 & 65,21 \\
\hline 51 a 70 FM & 200 & 32,3 & 83,07 & 115 & 18,26 & 46,95 & 65,21 \\
\hline$>70 \mathrm{FM}$ & 150 & 26,25 & 67,50 & 93,75 & & & \\
\hline
\end{tabular}

${ }^{a}$ Ingesta diaria estimada de pan. Se consideraron las porciones recomendadas para una alimentación saludable según las Guías Alimentarias Basadas en Alimentos para la población uruguaya (MSP, 2005).

'Los valores expresan los porcentajes de la Ingesta Adecuada (IA) de Na y Niveles Máximos (ULs) de Na, para no tener riesgos adversos (Dietary Reference Intakes for Water, Potassium, Sodium, Chloride, and Sulfate. 2004) que son cubiertas por la ingesta diaria estimada de pan para diferentes grupos de población y niveles de concentración de Na.

\section{FIGURA 2}

Estimación (\%) de la adecuación a las recomendaciones (Als) de Na con diferentes niveles de concentración de $\mathrm{Na}$ en el pan integral, en distintos grupos de población.

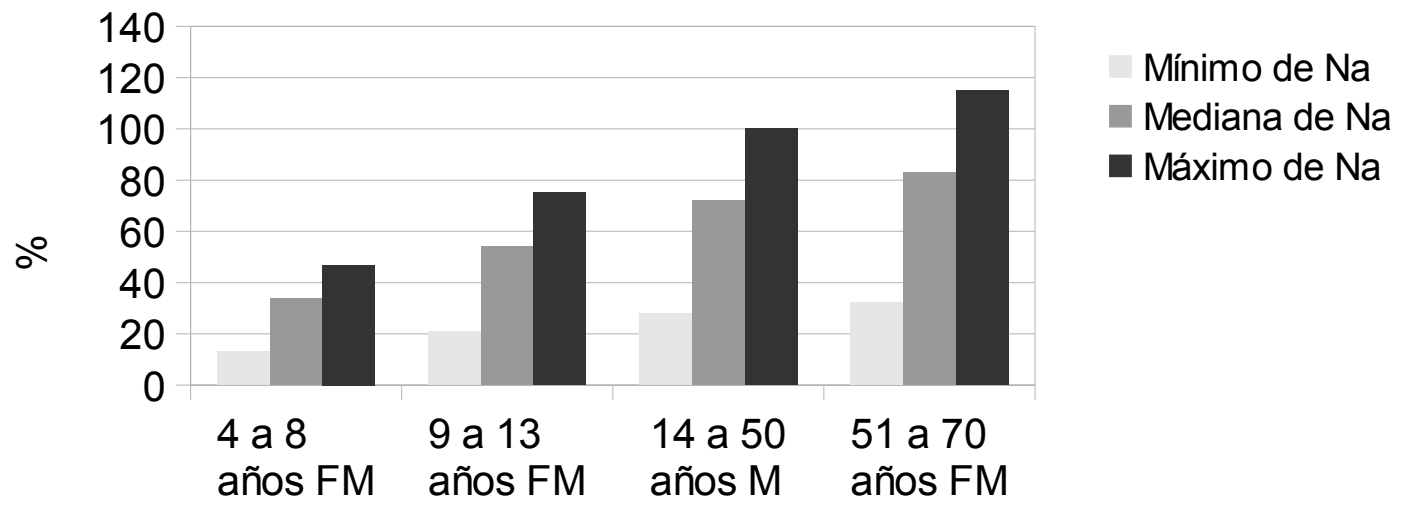


alimento fijado como meta, podría alcanzarse si se considera que el valor mínimo encontrado en el pan francés es menor al 50\% de esta meta (tabla 2 y3). Además sería posible de acuerdo a estudios de reducción de sal en la fermentación de la masa, poder desarrollar panes con buenas propiedades funcionales hasta con un $1,4 \%$ de sal sobre el total de harina, sin afectar la preferencia de los consumidores (6), porcentaje éste menor al planteado como meta en el acuerdo alcanzado por el Ministerio de Salud de Uruguay y CIPU. Al estudiar la adecuación a las ULs, en ningún caso se superan considerando ingestas de pan artesanal para diferentes grupos.

\section{Pan integral}

En relación a los lípidos en el pan integral artesanal de acuerdo a la declaración de los panaderos, se adiciona como materia grasa, aceite de girasol, grasa vacuna y margarina.
Estas materias grasas tienen diferente perfil lipídico lo que condiciona la calidad de lípidos del pan. La mediana de los lípidos (tabla 3) fue de 3,1, con un valor mínimo de 0,71 g a 4,75 g en $100 \mathrm{~g}$ de pan. Como lo permite la normativa para pan integral (5), el mismo puede tener materia grasa. Si bien la cantidad de lípidos es baja, el origen de los mismos es diverso, y es deseable el empleo de aceites vegetales, en detrimento de grasa vacuna, o margarinas hidrogenadas como fue declarado por las panaderías, pues esta calidad favorece un perfil dietario de riesgo para las ECNT, particularmente la $\operatorname{ECV}(3,15)$.

La mayoría de las panaderías de acuerdo a la declaración de los panaderos, cumplen con lo legislado en relación a la proporción de harina integral en la formulación del pan integral (mínimo 30\% de base seca harina integral) (5). La mediana fue de $50 \%$, pero hay importante variabilidad entre el valor

TABLA 6

Estimación (\%) de la adecuación a las recomendaciones (Als) de FDT con diferentes niveles de concentración de FDT en el pan integral, en distintos grupos de población.

\begin{tabular}{|c|c|c|c|c|}
\hline \multirow[t]{2}{*}{ Edad (años) y sexo } & \multirow[t]{2}{*}{$\operatorname{Pan}^{a}$ (g/día) } & \multicolumn{3}{|c|}{ Ingestab (g/día) } \\
\hline & & Mínimo & Mediana & Máximo \\
\hline 4 a $8 F M$ & 75 & 14,4 & 23,7 & 29,1 \\
\hline 9 a $13 M$ & 150 & 23,22 & 38,22 & 46,9 \\
\hline 9 a $13 \mathrm{~F}$ & 150 & 27,69 & 45,57 & 55,96 \\
\hline 19 a $50 M$ & 200 & 25,26 & 41,57 & 51,05 \\
\hline 19 a $50 \mathrm{~F}$ & 150 & 28,8 & 47,4 & 58,2 \\
\hline 51 a $70 M$ & 200 & 32 & 52,66 & 64,66 \\
\hline 51 a $70 \mathrm{~F}$ & 200 & 45,71 & 75,23 & 92 \\
\hline
\end{tabular}

angesta diaria estimada de pan. Se consideraron las porciones recomendadas para una alimentación saludable según las Guías Alimentarias Basadas en Alimentos para la población uruguaya (MSP, 2005).

'Los valores expresan los porcentajes de la Ingesta Adecuada (IA) de Na y Niveles Máximos (ULs) de Na, para no tener riesgos adversos (Dietary Reference Intakes for Water, Potassium, Sodium, Chloride, and Sulfate. 2004) que son cubiertas por la ingesta diaria estimada de pan para diferentes grupos de población y niveles de concentración de Na.

\section{FIGURA 3}

Estimación (\%) de la adecuación a las recomendaciones (Ais) de FDT con diferentes niveles de concentración de FDT en el pan integral, en distintos grupos de población.

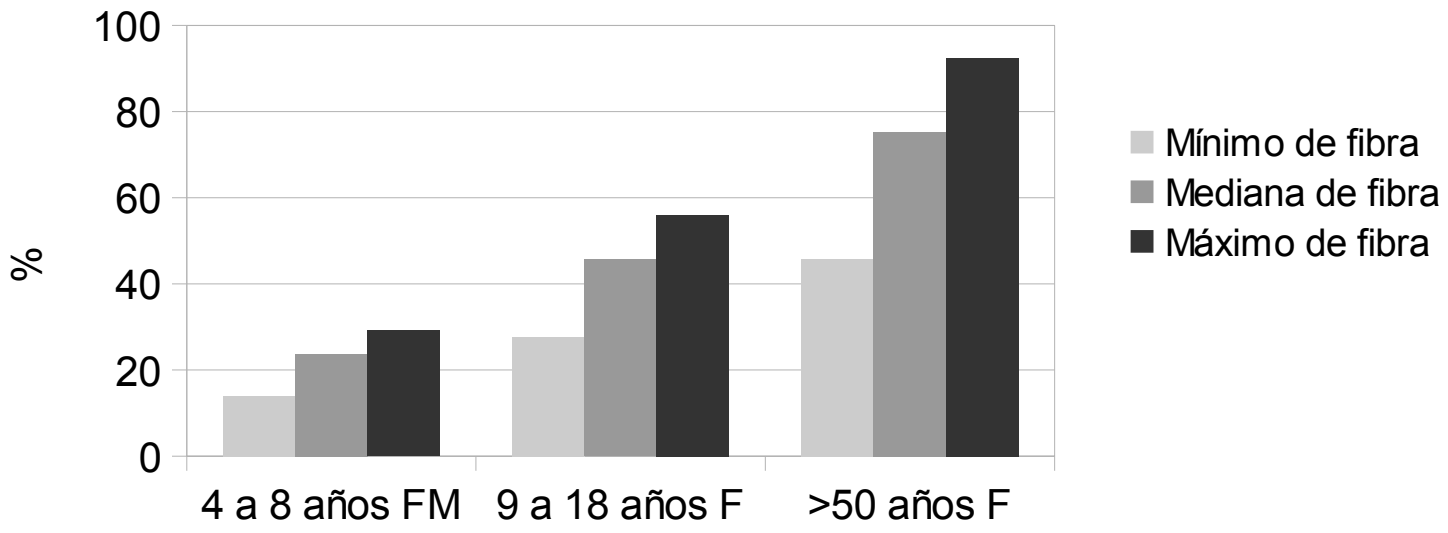


mínimo $27,3 \%$, ligeramente debajo del valor legislado y un $76,9 \%$ como máximo. Esto trae consecuencias que se reflejan en el rango de FD en las muestras de pan integral estudiadas.

Los porcentajes de IAs y ULs en $\mathrm{Na}$ en el pan integral (tabla 5, figura 2) son similares a los considerados para el pan francés; la concentración mínima de Na (tabla 3) en el pan integral, fue algo superior al pan francés (tabla 2). Por el contrario, al considerar el valor de la mediana y el máximo fue algo inferior al pan francés. Para cualquier grupo etario y sexo, no se superan las ULs, pero en personas desde los 9 años al consumir pan integral con el valor de la mediana identificado en este estudio (540 mg \%), sumado al consumo de otros alimentos y adición de sal en las preparaciones, se podría superar la IAs, ya que solo con porciones adecuadas de pan en el marco de una alimentación saludable se alcanza como mínimo el $50 \%$ de las IAs de Na. Del mismo modo que en el pan francés, es factible alcanzar con el resto de los alimentos consumidos en un día los $2 \mathrm{~g}$ de $\mathrm{Na}$ diarios o $5 \mathrm{~g}$ de sal establecidos por la OMS como límite máximo para una alimentación saludable (16).

Respecto al contenido de FDT en pan integral (tabla 6, figura 3), existen importantes diferencias en la contribución a la alimentación diaria al considerar las distintas concentraciones de FD según las muestras estudiadas (mínimo, mediana y máximo); esto se compadece con la variabilidad en la concentración de FDT en harina integral en las muestras estudiadas (tabla 1), a lo que se suma las diferencias en la formulación del pan. Con el valor mínimo encontrado de FDT, en niños desde los 9 años se cubre aproximadamente el 25\% de la IA y un $38 \%$ con el valor de la mediana; en el caso de personas mayores de 50 años el porcentaje es algo superior al 50\% si se considera el valor de la mediana de FDT.

Si se compara la cantidad de FDT del pan integral artesanal (tabla 3) con la cantidad declarada en la rotulación de panes integrales industrializados (12) se observa gran similitud en el rango y valores medios. Esto indica que es posible lograr con procesos estandarizados de elaboración a nivel artesanal un aporte dietario significativo de FDT a partir del consumo de pan integral artesanal. Del estudio se desprende la amplitud en las concentraciones de Na y FDT en pan francés e integral y la variabilidad en la calidad de la materia grasa empleada en el pan integral. En el caso del pan integral este estudio permite proponer un estándar en la formulación que permita alcanzar las máximas concentraciones de FDT. Además cobra importancia difundir a la población la composición de FDT dado el bajo consumo de cereales integrales, verduras y frutas en la dieta de los uruguayos (15). También es importante considerar el bajo aporte de materia grasa del pan integral y la exclusión de la misma en el pan francés, ya que hacen un diferencial positivo frente a otros productos panificados y de repostería de alto consumo en la población. Se debe alentar en la formulación en pan integral el empleo de aceites, que optimicen el perfil lipídico a favor de una dieta saludable, evitando grasa animal o hidrogenada vegetal con ácidos grasos trans. El $\mathrm{Na}$ es un constituyente altamente variable en los dos tipos de panes artesanales, pudiendo aportar una alta proporción de la IA en distintos grupos de la población considerando ingestas de pan acordes a una alimentación saludable.

En función de los resultados se concluye que con el consumo diario de pan francés e integral en una alimentación saludable se cubriría una importante proporción de las recomendaciones diarias de $\mathrm{Na}$ lo que podría constituir un riesgo para el desarrollo de ECV. Por lo tanto, es imperioso bajar las formulaciones de sal en el pan francés e integral artesanal.
Mientras que el pan integral sería un alimento que contribuiría en forma significativa al cumplimiento de las recomendaciones de FD para promover una alimentación saludable y contribuir a prevenir algunas ECNT en la población $(3,15,22)$.

Dentro de las limitaciones de este estudio se reconoce el restringido tamaño de la muestra, el cual no permite extrapolar los resultados al universo de panaderías del país. Existe por lo tanto la necesidad de ampliar el estudio con mayor número de panaderías y valorar el impacto del acuerdo interinstitucional de reducción de $\mathrm{Na}$ en el pan teniendo como base estos resultados así como estimular el desarrollo de formulaciones estandarizadas que permitan alcanzar los máximos niveles encontrados de FD y el agregado de materia grasa proveniente de aceites vegetales en el pan integral artesanal.

\section{RESUMEN}

La presente investigación evaluó la proporción de harina integral en pan integral con el nivel legislado; el contenido de lípidos y sodio ( $\mathrm{Na}$ ) en pan francés y lípidos, $\mathrm{Na}$ y fibra dietética total (FDT) en pan integral elaborado en panaderías de Montevideo y Canelones. Se estimó según cantidades diarias recomendadas el porcentaje de Na y FDT en relación a las Ingestas Adecuadas (Als) y los Límites Máximos Tolerables (Uls). El Na en pan francés cubre desde los 9 años un $64 \%$ de las IAs hasta cerca del 100\% en adultos desde los 51 años; el pan integral alcanza valores algo inferiores. La FDT cubre en niños de 9 años el 38\% de las IAs y 53\% en adultos mayores de 50 años. Este trabajo contribuye a disponer de una línea de base para el desarrollo de formulaciones con mínimos niveles de Na y óptimos de FDT en panes artesanales.

Palabras claves: Sodio; fibra dietética; Ingestas Adecuadas; Límites Máximos Tolerables, panes artesanales.

\section{REFERENCIAS}

1. WHO. Cardiovascular diseases. Main data. 2015. Available at: http://www.who.int/mediacentre/factsheets/fs317/es/ Last seen: 03/08/16.

2. Segunda Encuesta Nacional de Factores de Riesgo de Enfermedades Crónicas No Trasmisibles. 2013. Programa de Prevención de Enfermedades no Transmisibles. Departamento de Epidemiología. Ministerio de Salud Pública. [Second National Survey on Non-Communicable Chronic Diseases' Risk Factors. Non-Communicable Diseases Prevention Program. Epidemiology Department, Ministry of Health]. Available at: www.msp.gub.uy/.../2a -encuesta-nacionalde-factores-de-riesgo-de-enfer. Last seen: 28/02/2016.

3. WHO/FAO. Diet, Nutrition and the Prevention of Chronic Diseases. Report of the Joint WHO/FAO Expert Consultation. WHO Technical Report Series, No. 916 (TRS 916). Geneva, p.152, 2003.

4. Comisión Honoraria para la Salud Cardiovascular. Área Epidemiología y Estadística. Mortalidad por enfermedades cardiovasculares en el Uruguay [Honorary Commission for Cardiovascular Disease. Epidemiology and Statistics Area. Cardiovascular Disease Mortality in Uruguay], 2012. Available at:ttp://www.cardiosalud.org/ publicaciones/ mortalidad_2012.pdf. Last seen: 28/02/2016.

5. Reglamento Bromatológico Nacional (Uruguay). Decreto No. 315/994 de fecha 05/07/1994. $3^{a}$ ed. [National Bromatology Regulations (Uruguay). Decree No. 315/994, May 5, 1994. 3ed], Montevideo, IMPO, 2008, p. 460.

6. Zulueta L, Cervera V, Aja S. La dosis ideal de sal en el pan común. Evaluación en función de aspectos sensoriales, tecnológicos y nutricionales. Revista Profesional de Panadería 
y Pastelería [Recommended Salt Dosage in Common Bread. Assessment According to Sensory, Technological and $\mathrm{Nu}$ tritional Aspects. Bakery and Pastry Professional Journal], Spain 2010;1201-1202:8-12.

7. Valenzuela K, Quitaral V, Villanueva B, Zabala F, Atalah E. Evaluación del programa piloto de reducción de sal/ sodio en el pan en Santiago de Chile [Assessment of Salt/ Sodium Reduction Program in Santiago de Chile's Bread]. Chile Nutr J. 2013;40(2):119-122. Available at: http:// www.scielo.cl/scielo.php?script= sci_arttext \&pid=S071775182013000200004\&lang=pt. Last seen: 03/09/2015.

8. Lezcano E. Análisis de Producto. Productos panificados. Alimentos Argentinos. Ministerio de Agroindustria de la Nación [Bread Products. Argentinian Foods. The Nation's Ministry of Agro-Industry]. Available at: http://www. alimentos argentinos.gov.ar/contenido/sectores/farinaceos/ Productos/ProductosPanificados_2011_04Abr.pdf. Last seen: 20/08/ 2014

9. La Industria Panadera. Centro Industriales Panaderos del Uruguay [The Bread Industry. The Bakers Center of Uruguay]. Sept. 2013; 1096:6-7.

10. Pan American Health Organization. Salt-Smart Americas: $A$ Guide for Country-Level Action. Washington, DC: PAHO; 2013. [cited 201330 October]. Available at: http://www. paho.org/hq/index.php?option=com_docman\&task=doc_ view\&gid=21554\&Itemid, p. 30, 2013.

11. Morales C, Nieto A, Quiroga L, Quicazan M. Validación del método y determinación de fibra dietética soluble en harina de trigo y pan [Method Validation and Determination of Soluble Dietary Fiber in Wheat Flour and Bread]. Vitae. 2012; 19 (Suppl.1): s340-342.

12. González G., Pérez Castells L. Nutriguía: El libro de los alimentos y nutrientes. Cereales y derivados [The Book on Foods and Nutrients. Cereals and Byproducts], Montevideo, L\&M, 2016.

13. Bove MI, Cerruti F. Los alimentos y bebidas en los hogares: ¿un factor de protección o de riesgo para la salud y el bienestar de los uruguayos?. Encuesta nacional de gastos e ingresos de los hogares [Foods and Beverages at Home: A Protection or Risk Factor for the Wellbeing and Health of the Uruguayans? Household Income and Expenditures National Survey], 2005-2006. Montevideo, INE, 2008.

14. Taroco L. Estimación de los efectos de una disminución en el contenido de sal de los panificados sobre la salud cardiovascular [tesis de maestría] [Estimate of the Effect on Cardiovascular Health of Salt Content Reduction in Bread (master's degree thesis), Montevideo: Universidad Católica Dámaso Antonio Larrañaga [Catholic University Of Uruguay Damaso Antonio Larrañaga]; 2011. http:// magix.ucu.edu.uy:8086/opac9/servlet/edu.magix.bibopac. hbivedetreg?20041115,0,13.

15. MSP. Dirección General de la Salud. Programa Nacional de Nutrición. Grupo Interinstitucional de Trabajo para las Guías Alimentarias Basadas en Alimentos de Uruguay. Manual para la Promoción de Prácticas Saludables de Alimentación en la Población Uruguaya [Ministry of Health. National Health Directorate. National Nutrition Program. Inter-Institutional Work Group for Uruguay's Food-Based Alimentary Guidelines. Manual for the Promotion of Healthy Food Practices among Uruguay's Population], Montevideo. 2005.

16. WHO. Guidelines Sodium Intake in Children and Adults. Summary. 2013.

17. Institute of Medicine of de National Academies. Dietary Reference Intakes: Electrolytes and Water. Available at: https://fnic.nal.usda.gov/.../electrolytes_water.pdf. Last seen: 21/3/2016.

18. Jones Miller Julie. CODEX-Aligned Dietary Fiber Definitions Help to Bridge the 'Fiber Gap'. Nutrition Journal 2014;13:34. Available at: https://nutritionj. biomedcentral.com/.../1475-2891-1. Last seen: 28/08/2014.

19. Marlett JA, McBurney MI, Slavin JL. Position of the American Dietetic Association: Health Implications of Dietary Fiber. J Am Diet Assoc .2002; 102(7):993-1000.

20. Institute of Medicine, Food and Nutrition Board.Dietary Reference Intakes for Energy, Carbohydrate, Fiber, Fat, Fatty Acids, Cholesterol, Protein, and Amino Acids. (Macronutrients), 2005. Available at: http://www.nap.edu/ catalog.php?record_id=10490. Last seen: 20/08/2013.

21. Dilzer A. and cols. The Family of Dietary Fibers. Dietary Variety for Maximum Health Benefit. Nutr Food. 2003; 48(3):108-118.

22. Shewry PR, Hey S. The contribution of Wheat to Human Diet and Health. Food Energy Secur. 2015; 4(3):178-202. Available at: http://onlinelibrary.wiley.com/doi/10.1002/ fes3.64/full. Last seen: 15/4/2016. 